individuals. We aimed to describe sociodemographic characteristics of Chicago where POLs disseminated lupus-related information and to explore using the Area Deprivation Index (ADI) as a measure of the sociodemographic characteristics (higher ADI, more deprived).

Methods Fourteen POLs were recruited from primarily African American, underserved areas to disseminate lupus-related information. POLs recorded encounters throughout their social networks noting the number of individuals contacted and the addresses of venues where educational material was shared. GIS mapping documented the networks, the Healthy Chicago Data (HCD) described the sociodemographic factors and health resources of the POL networks, and GIS addresses linked geocodes with the ADI for these neighborhoods by census block tracks.

Results Nine POLs' social networks were concentrated in four selected predominantly African American communities (Washington Heights, Morgan Park, Englewood and Roseland) located on the Southside of Chicago where health, educational, and financial resources are limited using HCD. $8-17 \%$ of individuals living in these areas lacked health insurance, and the percent living below the Federal poverty line and for not completing high school was highest in Englewood $(30.8 \%$, $19.7 \%)$ and Roseland (27.2\%, 13.5\%), respectively. Five additional POLs also participated with social networks in other neighborhoods where we could calculate an ADI as shown in the figure 1. The percentage of ADI encounters in Chicago ranged from 9\% in ADI 1 to 18\% in ADI 10.

Conclusions POLs reached communities with varied ADI categories reflecting dissemination from least to most disadvantaged areas.

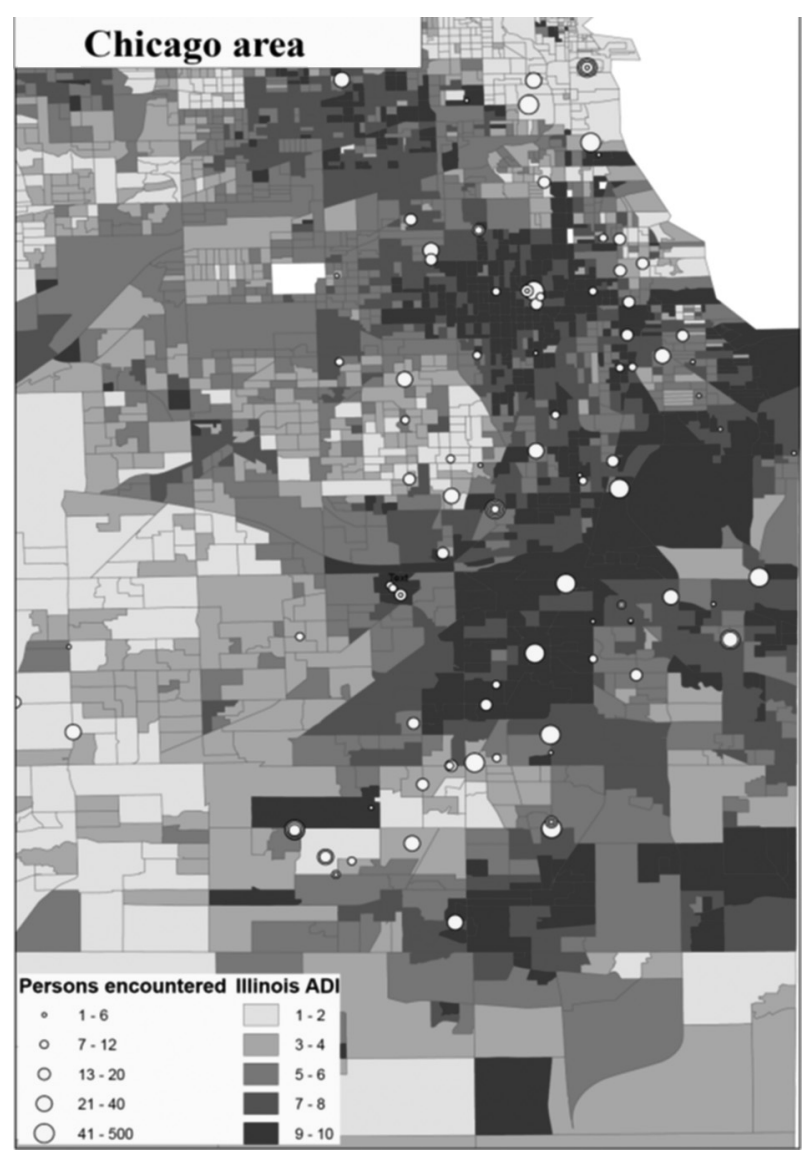

Abstract P66 Figure 1
Acknowledgements Funded by DHHS, OMH Grant \#1CPIMP171141-01-00.

\section{P67 JACCOUD'S ARTHROPATHY IN SYSTEMIC LUPUS ERYTHEMATOSUS: A CASE SERIES OF 52 BRAZILIAN PATIENTS}

Mittermayer Santiago. Rheumatology Service, HUPES/Federal University of Bahia, Salvador, Brazil

\subsection{6/lupus-2020-eurolupus. 112}

Background Jaccoud's arthropathy (JA) is a condition characterized clinically by 'reversible' joint deformities such as swan neck, thumb subluxation, ulnar deviation, 'boutonniere' and hallux valgus, along with an absence of articular erosions on a plain radiograph. JA was initially described in patients with rheumatic fever (RF), but presently the main clinical entity associated to JA is systemic lupus erythematosus (SLE). In general its prevalence in either SLE or RF is around 5\%.

The aim of the present study is to describe a series of SLE patients with JA, followed up at two referral lupus centers in Brazil. Methods The patients included in this study met the SLE classification criteria proposed by the American College of Rheumatology and also presented JA based on previously proposed criteria. Data collection was obtained through interviews, general physical examination with emphasis on the musculoskeletal system and review of medical records. The patients were originated from two SLE referral outpatient clinics in Salvador, Brazil and the search was conducted throughout 2018-2019.

Results Fifty-two patients with JA were included being 51 female, mean age was $48 \pm 13$ years and mean disease duration was $19 \pm 7$ years. The most frequently found joint deformities were swan neck [49/52 (94\%)]; ulnar deviation [32/52 (61\%)] and thumb subluxation [23/52 (44\%)].

Discussion To the best of our knowledge it is the largest case series of JA in SLE ever described. Some patients have been included in previous studies developed by our group. Based on the findings of such studies some conclusions can be drawn: 1) Although in the majority of the cases JA is a 'correctable' arthropathy sometimes the joints are fixed, resembling those seen in rheumatoid arthritis, even in the absence of bone erosions. 2) The level of interleukin 6 is higher in SLE patients with JA as compared to those without such complication. 3) Classically in JA no erosion is seen on plain radiographs, however its presence on magnetic resonance imaging or high-performance ultrasound examination is not so rare.

\section{P68 A DIET RICH IN WHEAT ALPHA-AMYLASE/TRYPSIN INHIBITORS (ATIS) ENHANCES DISEASE PROGRESSION IN THE MRL-FAS(LPR) MOUSE MODEL OF SYSTEMIC LUPUS ERYTHEMATOSUS}

\begin{abstract}
${ }^{1}$ Myriam Meineck, ${ }^{1}$ Simone Boedecker, ${ }^{2}$ Victor Zevallos, ${ }^{1}$ Andreas Schwarting, 1,3 Detlef Schuppan, ${ }^{2} J u$ lia Weinmann-Menke. 'Institute for Translational Immunology and Research Center for Immunotherapy (FZI), Univ. Medical Center, Johannes Gutenberg University Mainz, Mainz, Germany; ${ }^{2}$ Dept. of Nephrology, Johannes-Gutenberg University Mainz, Mainz, Germany; ${ }^{3}$ Division of Gastroenterology, Beth Israel Deaconess Medical Center, Harvard Medical School, Boston, USA
\end{abstract}

10.1136/lupus-2020-eurolupus.113

Background Wheat alpha-amylase/trypsin inhibitors (ATIs) are the second most prevalent proteins in wheat $(3-4 \%$ vs $80-$ 
$90 \%$ for gluten) and potent activators of the innate immune system via the toll like receptor 4 (TLR4)-MD2-CD14 complex in cells of the mononuclear phagocyte system (Junker Y et al, J Exp Med 2012), triggering several autoimmune/inflammatory diseases. In contrast, pure gluten that is de-enriched of ATIs shows no stimulatory activity. MRL-Fas(lpr) mice develop progressive and spontaneous glomerular, tubulointerstitial and perivascular kidney disease, arthritis, lymphadenopathy, splenomegaly and circulating autoantibodies in a syndrome that resembles systemic lupus erythematosus (SLE). Here we explored the effect of dietary ATIs on disease severity in MRL-Fas(lpr) mice.

Methods MRL-Fas(lpr) mice were placed either on a glutenfree diet (GFD) or a diet containing 25\% gluten (which contains amounts of ATIs equivalent to the human wheat based diet). We measured serum cytokines, proteinuria, haematuria, hemoglobinuria and histological and immunohistochemical markers of myeloid inflammation (F4/80 and CD68) in different affected organs (kidney, spleen and intestine).

Results Mice on a GFD showed lower levels of serum inflammatory cytokines (IL-6, KC and TNFa) that accompanied lower grade proteinuria, haematuria and hemoglobinuria during the study period. CD68, F4/80 and CD4 positive cells were also higher in the animals that consumed ATIs.

Conclusions Dietary wheat ATIs enhances SLE disease progression, in contrast, a GFD (which is ATI-free) had a protective effect on the development of SLE in MRL-Fas(lpr) mice, confirming the role of ATIs as important nutritional co-stimulant of inflammation in autoimmune diseases.

\section{P69 RISK FACTORS FOR ADVERSE PREGNANCY OUTCOME IN PATIENTS WITH SLE}

${ }^{1}$ Çiğdem Çetin, ${ }^{2}$ Tuğba Saraç-Sivrikoz, ${ }^{2}$ Müge Ateş-Tikiz, ${ }^{1}$ Yasemin Yalçınkaya, ${ }^{1}$ Ahmet Gül, ${ }^{1}$ Lale Öcal, 'Murat İnanç, ${ }^{2}$ ibrahim Kalelioğlu, 'Bahar Artım-Esen. 'Rheumatology Dept., Istanbul Faculty of Medicine, Istanbul University, Istanbul; '2Obstetrics and Gynaecology Dept., Istanbul Faculty of Medicine, Istanbul University, Istanbul, Turkey

\subsection{6/lupus-2020-eurolupus.114}

Objective Lupus pregnancies are considered as high risk because of both disease activation and pregnancy complications. In this study, we determined the risk factors associated with adverse pregnancy outcomes (APO) in pregnant patients with lupus followed up by both Rheumatology and Obstetrics and Gynaecology (O\&G) departments at our university.

Method 136 lupus patients with 168 pregnancies were analyzed. The course of pregnancies and fetal/neonatal prognosis were identified. Unexplained fetal death $\geq 12$ gestational weeks, neonatal death, preeclampsia, eclampsia, preterm birth due to HELLP and/or small for gestational age (SGA) infant were defined as APO. Clinical and laboratory findings, disease activity (SLEDAI-2K) and damage (SLICC/ACR) and conventional risk factors were compared between APO $(+)$ and APO (-) groups.

Results $71 \%$ of pregnancies resulted in live births and APOs occurred in 34\% (table 1). Renal and neuropsychiatric (NP) involvement, thrombocytopenia, antiphospholipid syndrome (APS), lupus anticoagulant and anti-cardiolipin $\operatorname{IgM}$ positivity rates were significantly higher in APO $(+)$ (table 2). Mean SLEDAI-2K during pregnancy and postpartum were higher in APO $(+)(2.2 \pm 3.6$ vs $1.2 \pm 2.04, \mathrm{p}<0.05 ; 4.9 \pm 6.03$ vs $2.7 \pm 5.01, \mathrm{p}=0.02$, respectively). There were significantly more patients with damage in the $\mathrm{APO}(+)$ and their mean damage score was significantly higher $(1.8 \pm 2.1$ vs $0.8 \pm$ $1.3, \mathrm{p}<0.05)$. The frequency of patients with NP, renal, cardiovascular and musculoskeletal damage was significantly higher in the $\mathrm{APO}(+)$ (figure 1).

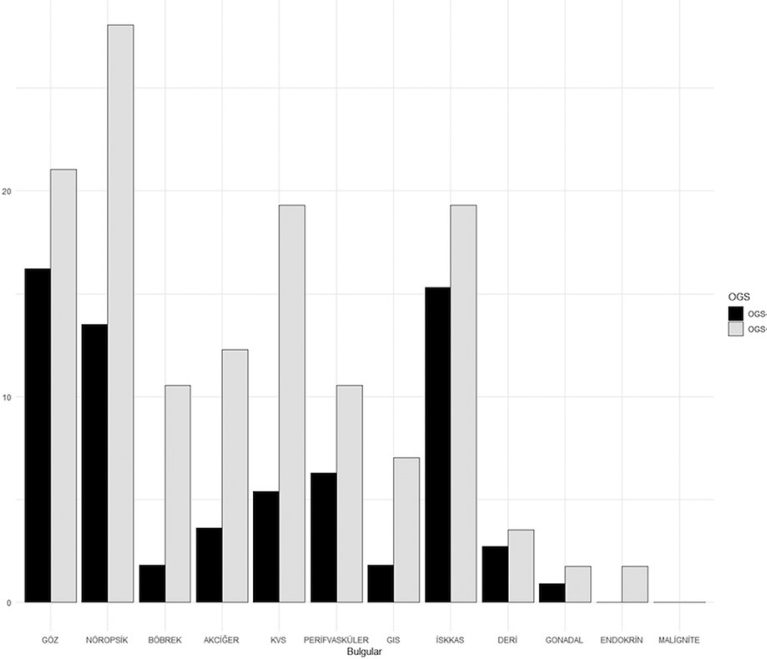

Abstract P69 Figure 1 Distribution of damage according to organs/ systems among APO (+) and APO (-) groups

Abstract P69 Table 1 Pregnancy $(n=168)$, fetal and neonatal outcomes in SLE pregnancy cohort $(n=136)$

\begin{tabular}{ll}
\hline & N (\%) \\
\hline Live birth & $119(70.8)$ \\
$\geq 22$ weeks still born & $11(6.5)$ \\
Neonatal Death & $1(0.6)$ \\
Maternal death & $1(0.6)$ \\
Preterm birth & $37(22)$ \\
SGA birth & $25(14.9)$ \\
Preeclampsia & $14(8.3)$ \\
- <32 weeks & $6(3.6)$ \\
- >32 weeks & $8(4.7)$ \\
HELLP & $5(3)$ \\
Intrauterine growth restriction (IUGR) & $23(13.7)$ \\
- <32 weeks & $13(7.7)$ \\
- >32 weeks & $10(6)$ \\
Pathologic uterine artery Doppler finding & $21(12.5)$ \\
Pathologic umbilical artery Doppler finding & $9(5.4)$ \\
Newborn 1st minute APGAR score <7 & $20(11.9)$ \\
Newborn 5th minute APGAR score <7 & $14(8.3)$ \\
Fetal cardiac disease & $13(7.7)$ \\
-Atrioventricular block & $7(4.1)$ \\
-Sinus bradycardia & $1(0.6)$ \\
-VSD & $2(1.2)$ \\
-VSD+ASD & $1(0.6)$ \\
-Aberrant right subclavian artery anomaly (ARSA) & $1(0.6)$ \\
-Mild hypertrophy of the left ventricle, stenosis in & $1(0.6)$ \\
pulmonary artery index & $13(7.7)$ \\
Fetal/neonatal congenital anomaly or disease & \\
(other than cardiac disease or conduction block & \\
\hline (IUGR: Intrauterine growth restriction, SGA: Small for gestational age) \\
\end{tabular}

